\title{
Improving air medical transport of the trauma patient from the ground
}

\author{
Joel Lockwood, MD; Alun Ackery, MD
}

Seriously injured patients often require transfer from a community hospital to a trauma centre, a hospital that is also an academic centre. Therefore, emergency medicine and general surgery trainees often participate in the air medical transport of trauma patients. The air ambulance process is recognized as a high-risk environment in which unstable patients are transported in resource-limited settings and poses a number of challenges. ${ }^{1}$ These include guiding management prior to transport, arranging interfacility transport, and coordinating medical management on arrival at the tertiary trauma centre.

Typically, the resident becomes involved as a remote trauma team member at the tertiary centre once the transfer decision and means of transport (rotary, fixed wing, or land) have already been determined. This is a challenging role for a number of reasons: geographic boundaries and time constraints affect communication; the scope of practice of the sending facility is variable and often not understood; and allied health professionals possessing varying scopes of practice are often independently involved in the active transport. With the addition of fatigue, stress, and other unidentified factors, a collaborative approach to patient care is often difficult.

As residents in emergency medicine, it is imperative that we understand how both community and academic hospitals manage trauma to facilitate transport between centres. This commentary uses a case-based approach to suggest strategies for residents to successfully coordinate air medical transport of trauma victims and improve collaboration between community and tertiary trauma physicians. Approaches include optimized communication, effective use of sending facility resources, and planning to maximize patient care during transfer.

\section{CASE}

A 23-year-old male, the sole passenger in a single rollover motor vehicle crash (MVC), was transported by emergency medical services to a suburban nontrauma hospital. On arrival, his heart rate was 110 beats/min and his blood pressure was 98/60 mm $\mathrm{Hg}$ with a respiratory rate of 20 breaths per minute and an oxygenation saturation of $99 \%$ on $10 \mathrm{~L} / \mathrm{min}_{2}$ via a nonrebreather facemask. He was agitated with a Glasgow Coma Scale (GCS) score of 9 (E3 V2 M4). Completion of the primary and secondary survey showed extensive bruising along the left side of his chest, subcutaneous emphysema, and an unstable pelvis. The emergency physician initiated resuscitation and decided that the patient required definitive treatment beyond the capabilities of the hospital. $\mathrm{He}$ contacted the closest designated trauma centre $(130 \mathrm{~km}$ away) and spoke with the emergency medicine resident on duty to coordinate transfer for definitive care while rotary air medical transport was being arranged.

\section{COMMUNICATION}

Telephone discussions between physicians involving the management of critically ill or injured patients are intrinsically difficult. Despite this, telecommunication remains the standard method of interaction used to coordinate interfacility air medical transport. Although there are a number of inherent challenges involved in a

From the Division of Emergency Medicine, Department of Medicine, University of Toronto, Toronto, ON.

Correspondence to: Dr. Alun Ackery, Department of Emergency Medicine, St. Michael's Hospital, 30 Bond Street, Room 1-008 Shuter Wing, Toronto, ON M5B 1W8; alun.ackery@mail.utoronto.ca.

This article has been peer reviewed. 
telephone conversation, including a lack of visual cues and contextualization, clear and concise information is possible and can be an effective aid in communication.

Ideally, both parties should be situated in areas free of distraction and interruption. Communication should proceed with clear, calm language that is free of slang and medical jargon and may not be universally understood.

Interaction should proceed in a courteous manner, and questions should be placed appropriately to avoid interruption. Contextualizing the patient's condition may be challenging over the telephone, and specific questions are often necessary. If something is unclear, ask why. With an MVC patient (as an example), perhaps the reason for delayed intravenous (IV) access was due to extensive burns over the extremities rather than unsuccessful attempts at a community centre (assumption of poor skills at a smaller centre). Digital photographs of injuries or radiographs can be sent rapidly through secured email to aid injury description, although patient confidentiality must be ensured. New technologies allow certain sites to make use of direct telemedicine communications for acute life-threatening conditions that are invaluable in improving real-time management. ${ }^{2}$

Following the initial interaction between physicians, it is important to summarize the case succinctly. Patient demographics, past medical history, trauma mechanism, identified injuries, and interventions undertaken should be clearly verbalized and acknowledged. It is important to present an itemized "to do" list prior to patient transport to accompany the patient (Appendix). Before completing the list, ask the sending physician if he or she requires anything else; it is the sending physician, after all, who remains responsible until the patient arrives at the trauma centre.

\section{UNDERSTANDING THE SENDING FACILITY}

To properly facilitate transfer and medical handover of a trauma patient, the receiving resident must have an understanding of the capacity of the sending facility and the experience of its staff. Depending on the situation, the sending facility could vary from a small nursing station with minimal resources to a large, wellstaffed suburban hospital with experienced emergency physicians. Knowing the resources and staff of the sending facility allows the accepting resident to better contextualize the clinical scenario. It will affect the transfer plan and should be tailored for each case.

\section{COLLABORATION}

The use of specialized trauma centres improves mortality and functional outcomes in injured patients. The extent to which intervention should be undertaken at the sending facility remains controversial and is likely situation dependent. ${ }^{3,4}$ In Canada, expansive geography means that patients requiring transfer will routinely be out of the optimal "golden hour" of transport. Consultation with trauma specialists can optimize early treatment and care.

During air transport, critically injured patients are transferred in physically restricted areas with limited human and physical resources. ${ }^{1}$ The goal of the sending facility should be to medically stabilize the patient before transport. While avoiding unnecessary delays in transport, they should anticipate and prepare for clinical changes that may arise due to positional shifts, altered physiology at altitude, and patient deterioration. For example, in a patient who may be ventilating and oxygenating well, anticipation of airway compromise during transport may necessitate endotracheal intubation prior to departure. It is important to remember that air trapped within any body cavity will expand as pressure decreases, mandating decompression prior to flight. Using the case described above, a chest tube must be placed in any confirmed or suspected pneumothorax.

Preparation for transport is best done as a collaborative effort between physicians at both sites. Material that may be required during transport, including fluids, blood, and medications, should be packed with the patient. Procedures that are difficult to perform in confined settings, including airway management, chest tubes, lines, and pelvic binding, are best done before patient departure.

It is the role of the receiving physician to advocate against unnecessary interventions or diagnostic tests that will delay transfer. Computed tomography (CT) has been shown to increase length of stay at the primary hospital by 90 minutes on average without contributing to definitive trauma management. ${ }^{5}$ These films are often incompatible with the software at other institutions and are not preferentially protocoled. To expedite transfer, we suggest only plain films of the chest, pelvis, and long bones to identify injuries that require rapid intervention prior to transfer.

\section{CASE SUMMARY}

The patient was hemodynamically stabilized and prepared for transport to the trauma centre for 
definitive care. After discussion with the receiving physician, the patient was intubated prior to transport. The focused assessment with sonography in trauma (FAST) examination was negative, and a pelvic binder was placed for a suspected open book pelvic fracture. A chest tube was placed in the left chest to manage a hemopneumothorax found on a chest $x$-ray. The patient received $2 \mathrm{~L}$ of $\mathrm{IV}$ crystalloid, $1 \mathrm{~g}$ of tranexamic acid, and 2 units of uncrossmatched packed red blood cells. Although the sending facility had the capabilities to do CT imaging, it was foregone to expedite transfer to a Level I trauma centre. An efficient handover was completed via telephone, and the patient was transported with two additional units of uncrossmatched blood, mannitol, copies of the chart, and the community hospital's trauma transport checklist (see the Appendix). The patient was in transit between hospitals for 45 minutes and met by the trauma team on arrival.

\section{CONCLUSION}

The implementation of complex trauma systems has necessitated increased collaboration between emergency physicians, traumatologists, and resident trainees working at different centres. This can be a stressful experience due to a lack of previous training, challenges with communication, and limited understanding of respective facilities and roles. A thoughtful approach is required to overcome these challenges and improve the efficiency of patient management throughout the transfer process.

Keywords: air transport, communication, resident, trauma

Competing interests: None declared.

\section{REFERENCES}

1. Singh JM, Macdonald RD, Bronskill SE, et al. Incidence and predictors of critical events during urgent air-medical transport. CMA7 2009;11:579-84.

2. Waite K, Silver F, Jaigobin C, et al. Telestroke: a multi-site, emergency-based telemedicine service in Ontario. 7 Telemed Telecare 2006;12:141-5, doi:10.1258/135763306776738611.

3. Nirula R, Brasel K. Do trauma centers improve functional outcomes: a national trauma databank analysis? 7 Trauma 2006;61:268-71, doi:10.1097/01.ta.0000230305.36456.4e.

4. MacKenzie EJ, Rivara FP, Jurkovich GJ, et al. A national evaluation of the effect of trauma-center care on mortality. $N$ Engl 7 Med 2006;354:366-78, doi:10.1056/NEJMsa052049.

5. Onzuka J, Worster A, McCreadie B. Is computerized tomography of trauma patients associated with a transfer delay to a regional trauma centre? C7EM 2008;10:205-8. 
APPENDIX: SUGGESTED CHECKLIST FOR AIR MEDICAL TRANSPORT OF THE TRAUMA PATIENT

\section{Airway/breathing}

Are there any indications for intubation? (patient oxygenating and ventilating, protecting airway, clinical course not anticipated to deteriorate)

- If the patient is intubated, has the endotracheal tube been secured at the appropriate depth?

Is necessary airway equipment being transported with the patient? (oropharyngeal/nasopharyngeal airways, oxygen tanks, bag-valve mask, facemasks, intubating supplies)

Has a portable chest $\mathrm{x}$-ray been completed?

- Does the patient have a pneumo-/hemothorax and require a chest tube?

- Has endotracheal tube placement been confirmed?

- Has central line placement been confirmed?

- Has chest tube placement been confirmed?

\section{Circulation}

Does the patient have adequate vascular access for resuscitation during transport?

- Are IV lines working, and will they be accessible?

- Are there enough IV lines if the patient deteriorates?

Does the patient require pelvic binding? (suspected unstable pelvic fracture on examination or pelvic $x$-ray)

Has a Foley catheter been placed to monitor resuscitation?

Have all compressible bleeding sites been controlled?

\section{Disability}

Are spinal precautions being maintained with a C-spine collar and backboard?

Has hypothermia been addressed?

- Has wet or cold clothing been removed. Is the patient dry?

- Are there adequate dry blankets for transport?

Have wounds been dressed/irrigated/splinted appropriately?

Will any of the following be required during transport?

Antibiotics

Fluids

Blood products

Analgesics

Tetanus prophylaxis

Mannitol/3\% $\mathrm{NaCl}$

Dextrose

Analgesia

Tranexamic acid

\section{Communication}

Has the appropriate physician been notified at the receiving hospital?

Will a written handover be sent with the patient? (confirmed injuries, interventions, and copies of laboratory tests/radiology) 